\title{
Surgical management and outcome of left ventricular pseudoaneurysm: our 11-year experience
}

\author{
Vaibhav Chugh ${ }^{1}$, Rahul Bhushan ${ }^{1}$, Narender Singh Jhajhria ${ }^{1}$, Manpal Loona ${ }^{1}$, Palash Aiyer ${ }^{1}$, Vijay Grover ${ }^{1}$, \\ Vijay Gupta ${ }^{1}$, Anubhav Gupta ${ }^{2}$ \\ ${ }^{1}$ Department of Cardiovascular and Thoracic Surgery, ABVIMS \& Dr. RML Hospital, New Delhi, India \\ ${ }^{2}$ Department of Cardiovascular and Thoracic Surgery, VMMC \& Safdarjung Hospital, Delhi, India
}

Kardiochirurgia i Torakochirurgia Polska 2021; 18 (4): 210-215

\begin{abstract}
Introduction: Left ventricular (LV) pseudoaneurysm is an uncommon condition with a high risk of death due to spontaneous rupture. The symptoms are nonspecific and diagnosis is often delayed. Surgical repair is the treatment of choice despite associated operative mortality.

Aim: Here we present a retrospective analysis of our experience in managing LV pseudoaneurysms over an 11-year period. Material and methods: Between May 2009 and April 2020, 7 patients ( 6 males and 1 female) with LV pseudoaneurysm underwent surgical repair at our center. Hospital records were accessed to obtain relevant clinical information and treatment outcomes. The mean age was 41.86 years (range: 7-73 years). Etiologies were post-myocardial infarction (4 patients) and prior endocarditis/pericarditis (3 patients). Pseudoaneurysms were posterobasal in 4 patients and apical in 3 patients. All the patients underwent surgical repair with resection of pseudoaneurysm and patch repair of the ventricular wall defect.

Results: All patients tolerated surgery well with no perioperative mortality or morbidity. Clinical condition and echocardiographic findings remained stable in all patients over their follow-up period (3 months to 3 years). Mortality occurred in a 73-year-old patient with post-MI posterobasal pseudoaneurysm, 15 months after surgery due to acute exacerbation of chronic obstructive pulmonary disease.

Conclusion: LV pseudoaneurysm is an entity that carries a high mortality risk. Timely diagnosis and early surgical intervention significantly improve the outcome.
\end{abstract}

Key words: left ventricular pseudoaneurysm, myocardial infarction, endocarditis.

\section{Introduction}

Left ventricular (LV) pseudoaneurysm is a contained cardiac rupture that is encircled by adherent pericardium or thrombus [1]. It is a rare complication that is reported in less than $0.1 \%$ of all myocardial infarction patients [2].

Myocardial infarction (MI) is the most common cause of this condition [3]. Other etiologies include cardiac surgery, trauma, and infection [4]. Studies have shown that LV pseudoaneurysm is mostly evident between 10 days and 2 months after MI [5].

Clinical manifestations are often nonspecific, presenting as congestive heart failure, chest pain, dyspnea, and arrhythmias. Echocardiography, computed tomography angiogram (CTA), and cardiac magnetic resonance (CMR) are good non-invasive imaging modalities for the diagnosis of pseudoaneurysm [1].

Untreated pseudoaneurysms of the left ventricle are extremely unstable and prone to rupture [6]. It is a cata- strophic condition leading to death in $48 \%$ of patients without surgical intervention [2]. Due to these factors, irrespective of chronicity, a timely diagnosis and early surgical treatment are recommended when LV pseudoaneurysm is detected $[7,8]$.

\section{Aim}

We aim to analyze retrospectively the outcome of surgical management of LV pseudoaneurysm in a single center.

\section{Material and methods}

This is a retrospective case series of seven patients with LV pseudoaneurysm operated on at our institute between May 2009 and April 2020. Patient records were analyzed for information regarding demographic data, clinical history, cardiovascular risk factors, etiology of pseudoaneurysm, imaging modalities used in establishing diagnosis and treatment outcomes. There were 6 males and 1 female

Address for correspondence: Palash Aiyer, Department of Cardiovascular and Thoracic Surgery, ABVIMS \& Dr. RML Hospital, New Delhi, India, e-mail: palashaiyer@gmail.com

Received: 5.05.2021, accepted: 5.09.2021. 
patient with a mean age of 41.86 years (range: $7-73$ years). The common symptoms were chest pain and dyspnea. One patient had a history of hypertension, 2 patients had diabetes and 3 patients had a history of smoking.

At our institution, all patients diagnosed with LV pseudoaneurysm underwent surgical treatment irrespective of the etiology and stage of disease presentation. All repairs were done via midline sternotomy under moderate hypothermic cardiopulmonary bypass (CPB) using aorto-bicaval cannulation with aortic cross-clamping and Del Nido cold blood antegrade cardioplegia. To prevent systemic embolization, the aorta was cross-clamped first and then dissection was done. Pseudoaneurysms were approached epicardially in most cases after dissecting surrounding pericardial adhesions. However, in three patients with submitral pseudoaneurysm having concomitant mitral pathology it was approached via the left atrium. After clot evacuation, the pseudoaneurysm neck was repaired by patch closure in all the patients (Figures 1-4).

We followed clinical parameters (e.g. good cardiac contractility, central venous pressure and hemodynamic stability) as a guide for weaning from CPB, instead of the principle of fixed reperfusion time ( one-third of cross clamp time) before weaning from CPB as followed at some centers. Primary closure of sternotomy was done in all cases. None of the patients needed mechanical circulatory support in the post-operative period. All patients were shifted from the operating room (OR) to the intensive care unit (ICU) on mechanical ventilation and inotropic support.

Patient 1: 7-year-old male patient who presented with dyspnea and CHF. He was diagnosed with a posterobasal submitral pseudoaneurysm $(2 \times 2 \mathrm{~cm})$ with healed vegetation on both mitral leaflets and severe mitral regurgitation $(M R)$ with normal left ventricular function. The diagnosis

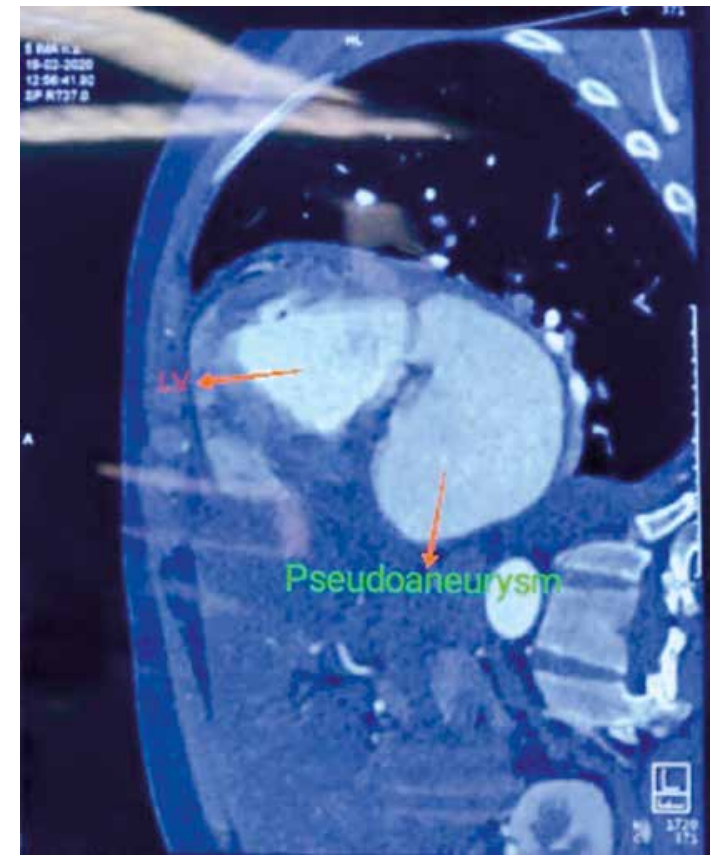

Figure 1. CT image depicting pseudoaneurysm arising from LV was confirmed by cardiac CT. The pseudoaneurysm was approached transatrially via Waterston's groove and the pseudoaneurysm neck was closed with a bovine pericardial patch. Mitral valve competence was restored by ring annuloplasty using the Carpentier Edward Physio annuloplasty ring (\#28).

Patient 2: 32-year-old male patient who presented with chest pain, dyspnea, fever with bilateral pleural effusion. Echocardiography was suggestive of normal functioning LV with pseudoaneurysm but could not delineate its origin. CT revealed a submitral pseudoaneurysm $(2 \times 1 \mathrm{~cm})$. The pseudoaneurysm was approached epicardially on the posterobasal aspect of the left ventricle and closed with a polytetrafluoroethylene (PTFE) patch and buttressed by the surrounding fibrotic pseudoaneurysm sac.

Patient 3: 17-year-old male patient who presented with recurrent fever, dyspnea, and arrhythmias. Echocardiography revealed vegetation along mitral annulus with large posterobasal submitral pseudoaneurysm $(6 \times 5 \mathrm{~cm}) \mathrm{com}$ municating into the LV through multiple openings with associated severe MR and normal LV function. CT and MRI showed multiple openings of the pseudoaneurysm into the left ventricle and left atrial appendage. The pseudoaneurysm was approached transatrially via Waterston's groove. One small opening immediately adjacent to the mitral annulus was closed by pericardial buttressed 4-0 prolene mattress sutures approximated to the mitral annulus and prosthetic valve suture ring. A second opening further down on the LV free wall was closed by an autologous pericardial patch. The third opening into the left atrial appendage was repaired by internal purse sting suture ligation of the appendage base. Mitral valve replacement was done using the number 25 St. Jude mechanical valve.

Patient 4: 73-year-old male patient, diagnosed with chronic obstructive pulmonary disease (COPD) and co-

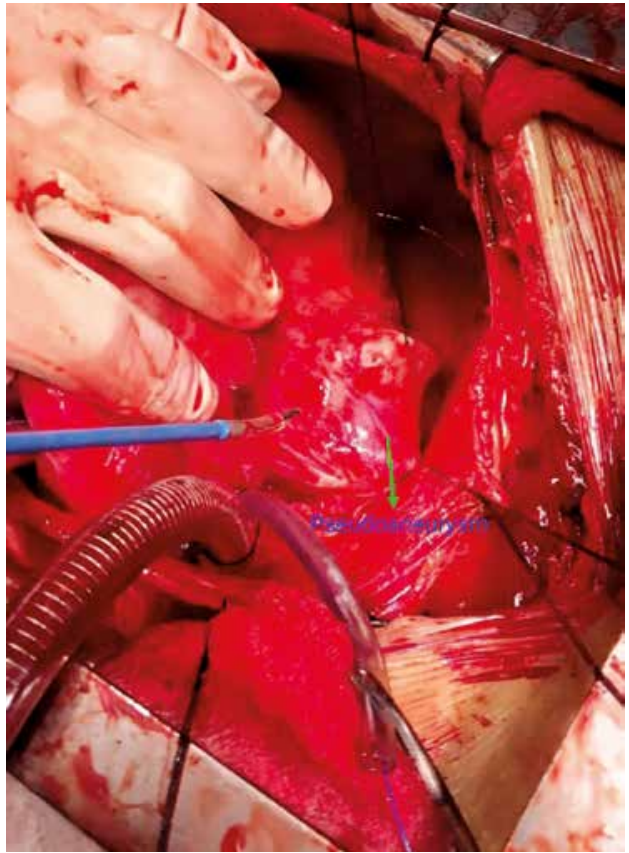

Figure 2. Intra-operative image showing pseudoaneurysm 


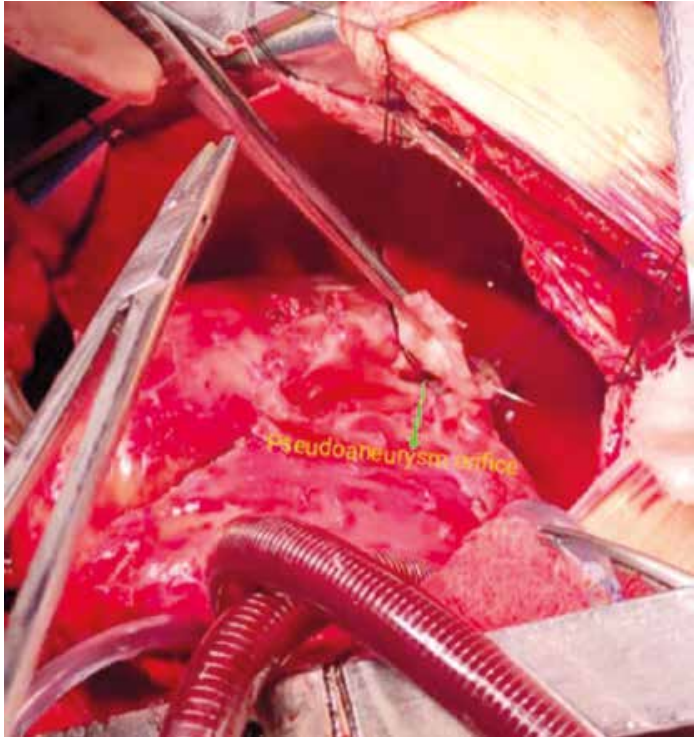

Figure 3. Intra-operative image showing pseudoaneurysm orifice

existing coronary artery disease with old inferior wall MI presented in CHF with dyspnea. Echocardiography showed posterobasal pseudoaneurysm $(5.5 \times 4.5 \mathrm{~cm})$ with severe MR with the LV function of $55 \%$. CAG showed single-vessel disease involving a posterior left ventricular artery (PLV) lesion. CT showed posterobasal LV pseudoaneurysm with moderate right pleural effusion and subsegmental collapse in the right upper lobe. Approached both via the LA (parallel to Waterston's groove) and epicardially. Pseudoaneurysm orifice was closed with a PTFE patch. Mitral valve replacement was done with a Carpentier Edward Perimount pericardial bioprosthetic $29 \mathrm{~mm}$ valve.

Patient 5: 62-year-old male patient diagnosed with triple vessel coronary artery disease (with mid-left anterior descending (LAD) artery and obtuse marginal (OM) tight lesion) and inferior wall $\mathrm{Ml}$ with coexisting hypertension and diabetes mellitus presented with chest pain. Echocardiography showed a hypokinetic inferior segment with the LV function of $45 \%$ and an apical LV pseudoaneurysm $(2.5 \times 2.5 \mathrm{~cm})$. Cardiac MRI suggested thinning of the LV wall with a focal inferior wall pseudoaneurysm. This pseudoaneurysm was approached epicardially and repaired with a Dacron patch and Teflon felt reinforcement. On pump coronary bypass was done and a venous graft was applied to the $\mathrm{OM} 1$ and $\mathrm{D} 1$.

Patient 6: 60-year-old female patient diagnosed with triple vessel coronary artery disease (with calcified LAD throughout its course) with prior anterior and inferior wall infarction. She had coexisting diabetes mellitus and hypertension. She presented with chest pain and dyspnea and was diagnosed on echocardiography with apical LV pseudoaneurysm $(3 \times 3 \mathrm{~cm})$ and ventricular septal defect (VSD) $(2 \times 2 \mathrm{~cm})$ with LV ejection fraction of $40 \%$. Cardiac MRI showed a thinned-out LV wall with pseudoaneurysm and VSD. Left ventriculotomy was done directly over the aneurysm. The visualized VSD was closed using an ePTFE patch with interrupted pledgeted 2-0 Ethibond sutures. The

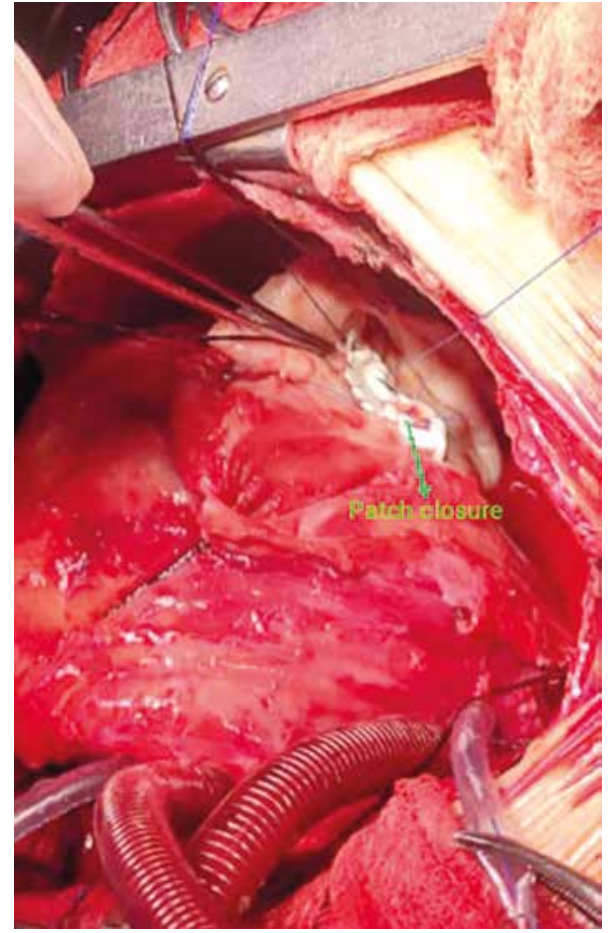

Figure 4. Intra-operative image depicting patch closure

left ventriculotomy was closed with 3-0 prolene in three layers (first interrupted mattress, second continuous mattress, third simple).

Patient 7: 42-year-old male patient diagnosed with triple vessel coronary artery disease (with LAD mid-cut-off) and coexisting hypertension and diabetes mellitus. He presented with chest pain and dyspnea and was diagnosed with LV apical pseudoaneurysm $(2.5 \times 2.5 \mathrm{~cm})$ with moderate LV dysfunction in echocardiography. The pseudoaneurysm was opened and the defect repaired. Repair was done in layers (first interrupted mattress, second continuous mattress, third simple) reinforced on Teflon felt and pericardium.

\section{Statistical analysis}

Data were analyzed using SPSS V26 software. The qualitative variables were expressed as percentages, while the quantitative variables were expressed as mean \pm 2 S.D.

\section{Results}

LV pseudoaneurysm occurred after transmural myocardial infarction in four out of 7 (57.14\%) cases. Etiology in $3(42.86 \%)$ cases was infective, of which 2 were post-infective endocarditis and 1 post-infective pericarditis. They were categorized as acute when diagnosed within two months of the index event and as chronic when diagnosed later than this. In our series four patients were acute as discovered incidentally by echocardiographic examination while they were in the hospital for fever with dyspnea and chest pain or acute myocardial infarction. The other three were chronic cases discovered during evaluation of their symptoms/signs at least 2 months after myocardial infarction, as determined 
Table I. Pre-operative characteristics of patients with left ventricular pseudoaneurysm

\begin{tabular}{|c|c|c|c|c|c|c|c|c|c|c|}
\hline Patient & Age & Sex & Etiology & Chronicity & Location & $\begin{array}{l}\text { Associated } \\
\text { cardiac lesion }\end{array}$ & LVEF & NYHA & EuroSCORE II & $\begin{array}{l}\text { Timing between } \\
\text { diagnosis and surgery }\end{array}$ \\
\hline 1 & 7 & M & $\begin{array}{l}\text { Infective } \\
\text { endocarditis }\end{array}$ & Acute & Posterobasal & MR & $55 \%$ & III & 2 & Early \\
\hline 2 & 32 & $M$ & $\begin{array}{l}\text { Infective } \\
\text { pericarditis }\end{array}$ & Acute & Posterobasal & - & $55 \%$ & III & 2 & Early \\
\hline 3 & 17 & M & $\begin{array}{c}\text { Infective } \\
\text { endocarditis }\end{array}$ & Acute & Posterobasal & MR & $50 \%$ & III & 2 & Early \\
\hline 4 & 73 & $M$ & $\mathrm{MI}$ & Chronic & Posterobasal & CAD, MR & $55 \%$ & III & 6 & Early \\
\hline 5 & 62 & $M$ & $\mathrm{MI}$ & Chronic & Apical & CAD & $45 \%$ & III & 2 & Early \\
\hline 6 & 60 & $\mathrm{~F}$ & $\mathrm{Ml}$ & Acute & Apical & CAD, VSD & $40 \%$ & IV & 13 & Urgent \\
\hline 7 & 42 & $M$ & MI & Chronic & Apical & CAD & $40 \%$ & III & 1 & Early \\
\hline
\end{tabular}

Table II. Postoperative data of patients with left ventricular pseudoaneurysm

\begin{tabular}{lcccccc} 
Patient & $\begin{array}{c}\text { Mechanical } \\
\text { ventilation time [h] }\end{array}$ & ICU stay [h] & $\begin{array}{c}\text { Hospital stay } \\
{[\text { days] }}\end{array}$ & $\begin{array}{c}\text { LVEF before } \\
\text { discharge }\end{array}$ & $\begin{array}{l}\text { NYHA } \\
\text { grade }\end{array}$ & Follow-up duration \\
\hline 1 & 12 & 36 & 3 & $55 \%$ & I & 18 months \\
\hline 3 & 12 & 36 & 3 & $55 \%$ & I & 3 months \\
\hline 4 & 12 & 36 & 5 & $55 \%$ & II & $\begin{array}{c}\text { Sudden death, } \\
15 \text { months }\end{array}$ \\
\hline 5 & 24 & 48 & 6 & $50 \%$ & II & 12 months \\
\hline 6 & 36 & 72 & 6 & $45 \%$ & II & 15 months \\
\hline 7 & 48 & 96 & 3 & $45 \%$ & 1 & 6 months \\
\hline
\end{tabular}

by the patients' histories. Pre-operative clinical features of the patients are presented in Table I.

The diagnosis was established using transthoracic echocardiography (TTE) routinely, with confirmatory contrast-enhanced computed tomography (CECT) of the chest, $\mathrm{MRI}$, and coronary angiography (CAG) done as required.

Pseudoaneurysm resection and closure of the underlying ventricular wall defect was performed in all patients. The mean cardiopulmonary bypass time was $183 \pm 63 \mathrm{~min}$ and the mean aortic cross-clamp time was $146 \pm 47 \mathrm{~min}$. Three (42.9\%) patients were diagnosed with concomitant severe MR. VSD resulting from ischemic rupture was present in $1(14.3 \%)$ patient. Concomitant coronary artery disease (CAD) was noted in 4 (57.1\%) patients with single-vessel disease in one and multiple vessel involvement in 3 cases.

The ventricular wall defect giving rise to pseudoaneurysm in our series was posterobasal in 4 (57.1\%) patients and apical in 3 (42.9\%) patients.

The patch materials used were PTFE and pericardium each in 3 patients, and terephthalate (Dacron) in 1 patient.

One patient with post-infarction ventricular septal defect was repaired using an ePTFE patch. Mitral regurgitation was seen in $3(42.9 \%)$ patients, of which one was treated by annuloplasty ring and the other two by valve replacement. Only one of the four having CAD underwent concomitant coronary revascularization as in the others the LAD target was non-graftable or the target territory had good collateralization.
Mean hospital stay was 4 days. The postoperative outcome was uneventful in all patients. An echocardiogram was done on the third or fourth postoperative day to assess cardiac function and confirm obliteration of pseudoaneurysm. The ventricular function was either improved or unchanged after repair as confirmed by the echocardiogram. The mean follow-up time was 15 months (3 months to 3 years). All the patients were in NYHA grade I or II during follow-up. Death occurred in one patient after 15 months due to acute exacerbation of COPD. Post-operative characteristics of the patients are summarized in Table II.

\section{Discussion}

LV pseudoaneurysm is a rare condition which results from free wall rupture contained by adjacent pericardium [8]. The most common cause of left ventricular pseudoaneurysm (55\%) is transmural myocardial infarction. Less frequent causes include complications of cardiac surgery (33\%), chest trauma (7\%), endocarditis (5\%), and, rarely, as a consequence of purulent pericarditis or tumor infiltration [9]. In our study, 4 (57.1\%) cases of LV pseudoaneurysm occurred following myocardial infarction. Two (28.6\%) cases developed as a complication of infective endocarditis while one occurred after infective pericarditis. Both of these etiologies have been reported previously in the literature, with the latter being very rare $[10,11]$. Patients with LV pseudoaneurysm usually present within 2 months of myocardial infarction [2]. In our series four patients presented within 
2 months of infarction/infection (acute) and three patients later than 2 months (chronic).

In myocardial infarction, the weakened LV wall occasionally ruptures. If contained within the surrounding pericardium, the resulting hematoma organizes into fibrous tissue and forms a saccular structure communicating with the ventricular chamber via a narrow neck $[12,13]$. The pathogenesis behind the development of pseudoaneurysm in infective cases is that bacterial invasion into the annulus of the valve leads to tissue destruction, necrosis, and abscess formation. Spontaneous drainage of the abscess into the aorta or left ventricle then results in the formation of the pseudoaneurysm [10]. Another theory is that infection may cause weakening of the wall, predisposing to dissection and cavity formation, and finally resulting in a pseudoaneurysm [10].

Mostly the pseudoaneurysm cavity communicates with heart through a single opening but multiple communications, while rare, have been reported [14]. In our series, 1 patient with post-endocarditis submitral giant pseudoaneurysm had three different openings leading into the pseudoaneurysm cavity.

Post-infarction pseudoaneurysms have been reported to originate usually at the posterior basal and sometimes at the apical segment of the left ventricle after occlusion of the right coronary or left anterior descending artery [9]. In our case series, three pseudoaneurysms were due to LAD occlusion while one was due to PLV occlusion. Submitral pseudoaneurysms are known to occur following prior valve surgery or endocarditis, as was seen in our series.

Most patients with LV pseudoaneurysms are asymptomatic [3]. Signs and symptoms, when present, are attributable to congestive heart failure or arrhythmias and are thus neither sensitive nor specific for establishing the diagnosis. TTE/TEE is usually adequate for the diagnosis and follow-up [3]. In our series, LV pseudoaneurysm was diagnosed by TTE in all cases, except one, which was misdiagnosed as descending thoracic aortic aneurysm, thus necessitating further CT study. Additional imaging modalities including LV angiography, chest CECT, cardiac CT, and CMR are valuable adjuncts to differentiate true from false aneurysms [2]. Cardiac MRI and CECT are very useful in identifying anatomic detail and planning surgery.

Untreated pseudoaneurysm has a $30 \%$ to $45 \%$ risk of rupture as it is communicating freely to the left ventricle and tends to expand. If ruptured, this can lead to cardiac tamponade, shock and death $[6,15]$. March et al. reported that the dynamics of collagen breakdown and subsequent resynthesis following infarction or other inflammatory processes play a major role in determining wall strength and thus determining the susceptibility of LV pseudoaneurysm to rupture [16]. The study states that significant collagen breakdown occurs with proteolysis of the myocardium and is associated with an influx of polymorphonuclear leukocytes (PMN) and macrophages into the infarct region thus leading to cardiac rupture. Another theory suggested is shearing of intramyocardial blood vessels, resulting in in- tramural hematoma and dissection, thus predisposing to cardiac rupture [16]. Therefore surgery is indicated for all patients as soon as the diagnosis is established, irrespective of symptoms and chronicity $[12,15]$. In our study, all the patients underwent surgical repair of the pseudoaneurysm.

After the evacuation of the clot, the ventricular wall defect can be repaired either by primary suture closure or patch closure using the synthetic, autologous, or bovine pericardial patch. Patch closure is preferred over direct closure in large posterobasal defects - to preserve mitral valve geometry and to avoid pseudoaneurysm recurrence due to suture dehiscence [15]. However, no definitive recommendation on type of patch to be used in different etiologies is available. Recently, the sutureless technique using fibrin glue to anchor the patch has been used in acute forms of post-MI left ventricular pseudoaneurysms; however, there is concern regarding recurrent pseudoaneurysm and further evidence is required [17]. We performed patch closure in all our patients. PTFE and a pericardial patch were used in three patients each while a Dacron patch was utilized in one patient. The one patient with submitral pseudoaneurysm from our series had multiple openings in the wall leading to the pseudoaneurysm cavity. So, we performed patch closure of the larger posterior wall defect and direct suture repair of the smaller juxta-annular defect. We preferred to use autologous pericardium as a preferred patch in patients with infective etiology to minimize the presence of foreign tissue. However, in patients with infective pericarditis, the native pericardium was not available due to being involved in the pathology so a PTFE patch was utilized. In the rest of the patients, the type of patch used was totally dependent on the surgeon's choice and availability.

The management of patients with cardiac pseudoaneurysm is frequently complicated by comorbidities such as coronary artery disease and heart failure. Relatively poor prognosis has been noted in those caused by myocardial infarction and in patients requiring more complex surgery (e.g., concomitant pseudoaneurysm and valvular replacement) [15]. In our study, mitral valve surgery was performed in three patients and coronary revascularization in one patient. All these patients recovered without developing any morbidity in the post-operative period. Mean hospital stay in our study was 4 days, which is shorter than in other studies [15]. This is because ours is a small functioning unit with limited bed capacity but a relatively large patient volume, leading to a high turnover rate. So, barring one patient needing urgent surgery, all other patients were thoroughly evaluated on an outpatient basis and admitted directly for planned early surgery. In the post-operative period, once the patients had been transferred from the ICU to the ward and remained clinically stable with acceptable echocardiogram parameters, we discharged them and called for the first follow-up early within a week.

The overall early mortality rate reported for LV pseudoaneurysm in some initial published series was in the range of $20-35 \%[3,15,18,19]$. Improvements in surgical technique have led to a decrease in mortality rate to as low 
as less than $10 \%$ [20]. All of our seven cases of pseudoaneurysm were surgically repaired without any peri-operative deaths. Similarly, in the study conducted by Perek et al., all 8 patients survived the surgery and none required reoperation [21].

Ours is a retrospective study with a small sample size and no control population to compare the results with. The follow-up period is short in our study as patients were lost to follow-up in the due course of time. This is because most of the patients have a poor socio-economic background and are not well educated in our part of the world. Expenditures for seeking medical attention (such as transportation charge or investigation cost) act as a strong deterrent, especially when the patient feels clinically relieved and asymptomatic in the follow-up period.

\section{Conclusions}

It is challenging to diagnose LV pseudoaneurysm because of the ambiguity in its presentation; thus, diagnosis in a timely manner is crucial. A high index of clinical suspicion and routine echocardiographic screening for detection of LV pseudoaneurysm are essential to improve the outcome in these patients. Early surgical repair is required to reduce the risk of rupture and improve long-term survival with good functional capacity.

\section{Disclosure}

The authors report no conflict of interest.

\section{References}

1. Bisoyi S, Dash AK, Nayak D, Sahoo S, Mohapatra R. Left ventricular pseudoaneurysm versus true aneurysm a diagnosis dilemma. Ann Card Anaesth 2016; 19: 169-72.

2. Alapati L, Chitwood WR, Cahill J, Mehra S, Movahed A. Left ventricular pseudoaneurysm: a case report and review of the literature. World J Clin Cases 2014; 2: 90-93.

3. Eren E, Bozbuga N, Toker ME, Keles C, Rabus MB, Yildirim O, Guler M, Balkanay $M$, Isik $O$, Yakut C. Surgical treatment of post-infarction left ventricular pseudoaneurysm: a two-decade experience. Tex Heart Inst J 2007; 34: 47-51.
4. Ikeda N, Yasu T, Kubo N, Hirahara T, Sugawara Y, Kobayashi N, Hashimoto S, Tsuruya $Y$, Fujii M, Saito M. Effect of reperfusion therapy on cardiac rupture after myocardial infarction in Japanese. Circ J 2004; 68: 422-426.

5. Csapo K, Voith L, Szuk T, Edes I, Kereiakes DJ. Postinfarction left ventricular pseudoaneurysm. Clin Cardiol 1997; 20: 898-903.

6. Villanueva C, Milder D, Manganas C. Ruptured left ventricular false aneurysm following acute myocardial infarction: case report and review of the literature. Heart Lung Circ 2014; 23: e261-3.

7. Sheikh WR, Sehgal P, Verma A, Haldar M, Jaiswal S. Left ventricular pseudoaneurysm post myocardial infarction. Int J Crit Illn Inj Sci 2019; 9: 43-45.

8. Garrido JM, Ferreiro A, Rodríguez-Vázquez JF, Prada P, Verdugo S, Silva J, López-Checa S, Sánchez-Montesinos I. Left ventricle postinfarction pseudoaneurysm: anatomical forms and surgical management. Surg Sci 2014; 5: 138-145.

9. Faustino M, Ranchordás S, Abecasis J, Freitas A, Ferreira M, Gil V, Morais C, Neves JP. Left ventricular pseudoaneurysm - a challenging diagnosis. Rev Port Cardiol 2016; 35: 373e1-373e6.

10. Shimotakahara J, Hirata K, Nakazato J, Yagi N, Takahashi T, Wake M, Yasumoto $\mathrm{H}$, Tengan $\mathrm{T}$, Mototake $\mathrm{H}$. Left ventricular pseudoaneurysm as a complication of prosthetic mitral valve infective endocarditis. J Cardiol Cases 2013; 8: e27-e30.

11. Moraes AN, Ferreira AG Jr, Ferreira SM. Left ventricular pseudoaneurysm complicating infective pericarditis. Heart 1999; 82: 393-394.

12. Jacob JL, Buzelli G, Machado NC, Garzon PG, Garzon SA. Pseudoaneurysm of left ventricle. Arq Bras Cardiol 2007; 89: e1-e2.

13. Fialho G, Fischer C, Cordovil A, Filho O. Chronic left ventricular pseudoaneurism of undetermined etiology. Arq Bras Cardiol 2010; 94: e4-e6.

14. Mahilmaran A, Nayar PG, Sheshadri M, Sudarsana G, Abraham KA. Left ventricular pseudoaneurysm caused by coronary spasm, myocardial infarction, and myocardial rupture. Tex Heart Inst J 2002; 29: 122-125.

15. Atik FA, Navia JL, Vega PR, Gonzalez- Stawinski GV, Alster JM, Gillinov AM Svensson LG, Pettersson BG, Lytle BW, Blackstone EH. Surgical treatment of postinfarction left ventricular pseudoaneurysm. Ann Thorac Surg 2007; 83: 526-531.

16. March KL, Sawada SG, Tarver RD, Kesler KA, Armstrong WF. Current concepts of left ventricular pseudoaneurysm: pathophysiology, therapy, and diagnostic imaging methods. Clin Cardiol 1989; 12: 531-540.

17. Sakaguchi G, Komiya T, Tamura N, Kobayashi T. Surgical treatment for postinfarction left ventricular free wall rupture. Ann Thorac Surg 2008; 85: 1344 1346.

18. Frances C, Romero A, Grady D. Left ventricular pseudoaneurysm. J Am Coll Cardiol 1998; 32: 557-561.

19. Pretre R, Linka A, Jenni R, Turina MI. Surgical treatment of acquired left ventricular pseudoaneurysms. Ann Thorac Surg 2000; 70: 553-557.

20. Hulten EA, Blankstein R. Pseudoaneurysms of the heart. Circulation 2012; 125: 1920-1925.

21. Perek B, Jemielity M, Dyszkiewicz W. Clinical profile and outcome of patients with chronic postinfarction left ventricular false aneurysm treated surgically. Heart Surg Forum 2004; 7: E132-5. 